\title{
Breaking Barriers of FIB-SEM for Large Volume Connectomics and Cell Biology
}

\author{
C. Shan $\mathrm{Xu}^{1}$, Kenneth J. Hayworth ${ }^{1}$, Song Pang ${ }^{1}$, Zhiyuan $\mathrm{Lu}^{2}$, and Harald F. Hess ${ }^{1}$ \\ 1. Janelia Research Campus, Howard Hughes Medical Institute, Ashburn, United States. \\ 2. Department of Psychology and Neuroscience, Dalhousie University, Halifax, Canada.
}

Focused Ion Beam Scanning Electron Microscopy (FIB-SEM), developed for materials science and the semiconductor industry, has been applied to biological imaging for over a decade [1]. Conventional FIBSEM systems offers superior isotropic resolution, yielding data that needs minimal image registration and post processing. However, deficiencies in its imaging speed and long-term system stability limit the maximum imaging volume. I will present advancements that accelerate image acquisition and markedly improve reliability of conventional FIB-SEM, expanding the imageable volume by more than four orders of magnitude to at least $10^{7} \mu \mathrm{m}^{3}$ at $8 \times 8 \times 8 \mathrm{~nm}^{3}$ voxel resolution [2]. These large volumes are ideal for connectomics, where the excellent $\mathrm{z}$ resolution can help in tracing of small neuronal processes of small insects and minimize the time-consuming human proofreading effort. Even higher resolution are achievable on smaller volumes for cell biology studies, where an entire mammalian cell can be imaged at $4 \times 4 \times 4 \mathrm{~nm}^{3}$ voxel resolution.

Many modalities of electron microscopy (EM) can probe cellular structure at the nanometer scale. However, despite considerable progress over the past decade in developing high-resolution 3D imaging, there remain important limitations, reflecting an inherent trade-off between resolution and the size of the 3D volume. The different EM methods now available and their advantages and disadvantages have been reviewed recently. The graphical summary (Figure 1), which shows the operating regimes of the different EM methods in terms of sample volume and minimum isotropic resolution, identifies an important region of resolution-volume space that remains inaccessible with current techniques. For demanding applications such as tracing neuronal processes in three dimensions, high resolution in the $\mathrm{z}$ axis, in addition to the xy plane, is critical. FIB-SEM offers exactly this capability, with $\mathrm{x}, \mathrm{y}$, and $\mathrm{z}$ resolution all $<10 \mathrm{~nm}$, in addition to other significant advantages, such as superior registration and fully automated operation. However, to date the FIB-SEM approach has seldom been used in neuroscience because of its severe volume limitation, dictated by the limited imaging speed and the limited duration of smooth and consistent ablation, typically resulting in an imaged volume of less than the extent of a single neuron. Because the process is destructive, in order to apply FIB-SEM to connectomics, one has to advance the technique to be error-free in the ablation-imaging cycle, with virtually perfect continuity and consistency.

To meet the large volume demands of connectomics, we designed a customized FIB-SEM system to specifically address the deficiencies in conventional FIB-SEM so that one can image faster and longer. These technological advances include: 1) imaging speed improvements; 2) error detection of all possible failures; and 3) seamless recovery. Our enhanced FIB-SEM system empowers researchers to explore a broad variety of applications, ranging from large volume connectomics to high resolution cell biology. The application space can be split into three operating regimes: Standard Resolution, High Resolution, and High Throughput (at reduced resolution). Examples from Drosophila brain, mammalian neural tissue, and cultured mammalian cells datasets will be presented to illustrate the power of isotropic resolution and large imaging volume. 
References:

[1] JA Heymann et al, J. Struct. Biol. 155 (2006), 63-73.

[2] CS Xu et al, Elife 6 (2017), p. e25916.

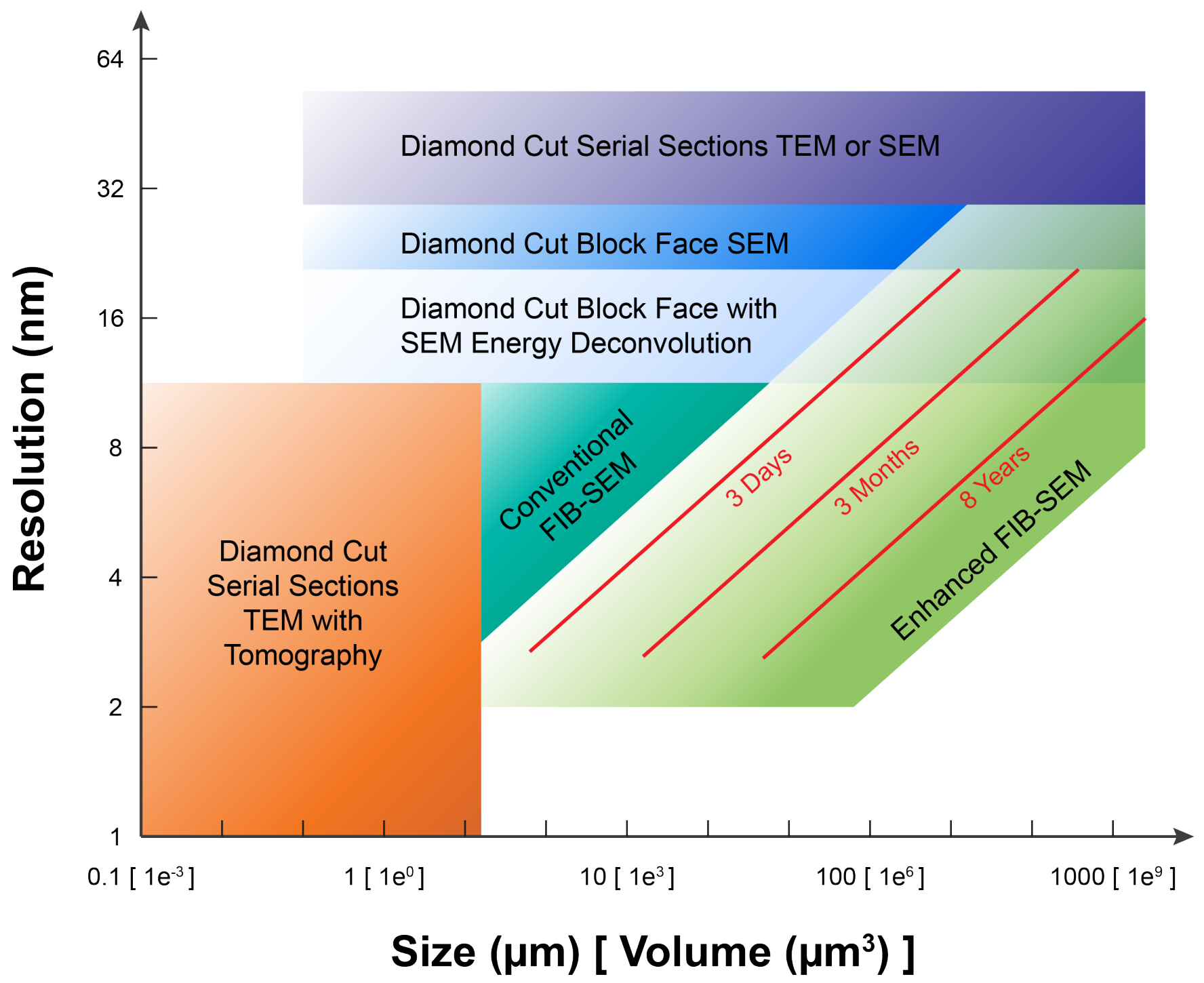

Figure 1. A comparison of various 3D imaging modalities in the application space defined by resolution and total volume. 